\title{
Chirurg in humanitärer Mission
}

\author{
Eine halbe Million Kinder, vorwiegend aus Afrika und Asien, sterben jährlich an \\ Krankheiten, die geheilt werden könnten. Zusammen mit Terre des hommes - \\ Kinderhilfe (Tdh) kämpft der Herz- und Gefässchirurg Ludwig K. von Segesser gegen \\ diese traurige Bilanz an.
}

Hanni Mathis

Terre des hommes - Kinderhilfe

Korrespondenz: Hanni Mathis

Terre des hommes - Kinderhilfe Schwarztorstrasse 20 CH-3007 Bern

Tel. 0586110790

rgf@tdh.ch
Vor knapp 50 Jahren rief Terre des hommes - Kinderhilfe ein bis heute schweizweit einzigartiges Projekt ins Leben: «Voyage vers la vie». In dieser Zeit konnte bereits der beachtlichen Anzahl von über 10000 Kindern geholfen werden. Das Projekt soll dazu beitragen, das medizinische Wohlstandsgefälle der Welt zu überbrücken. Denn wie der Reichtum ist auch die medizinische Versorgung ungleich verteilt. So steht in Europa für eine Million Menschen ein Herzchirurg zur Verfügung, in Asien beträgt das Verhältnis jedoch 1:16 Millionen und in Afrika gar 1:33 Millionen Menschen. Und die Kluft zwischen Europa und den Drittweltländern wird immer grösser.

Gemäss UNO-Kinderrechtskonvention hat jedes Kind nicht nur das Recht auf medizinische Grundversorgung, sondern auch auf eine auf sein Leiden zugeschnittene Spezialbehandlung. So diagnostiziert und behandelt Tdh in aktuell neun Einsatzländern (Benin, Guinea, Irak, Madagaskar, Marokko, Maureta- nien, Senegal, Togo und Tunesien) Kinder mit medizinischen Spezialproblemen.

\section{Ein Projekt, das nachhaltig wirkt}

Wenn immer möglich werden die Kinder vor Ort in Zusammenarbeit mit lokalen Spitälern operiert. Falls eine Operation im Projektland selbst nicht möglich ist - aufgrund von fehlenden Geräten oder mangels Know-how - werden die Patienten nach Europa überwiesen. Professor von Segesser vom Lausanner Universitätsspital (CHUV) ist einer der Chirurgen, die Kinder aus Drittweltländern in der Schweiz behandeln. Die Auswahl der Patienten erfolge sehr gezielt, erklärt er das Prozedere. Die Eltern müssen mit der Massnahme einverstanden sein, und es werden nur Familien berücksichtigt, die sich eine Operation nicht selbst leisten können.

Eine weitere Achse des Projekts beinhaltet den Wissensaustausch zwischen der Schweiz und den Pro-

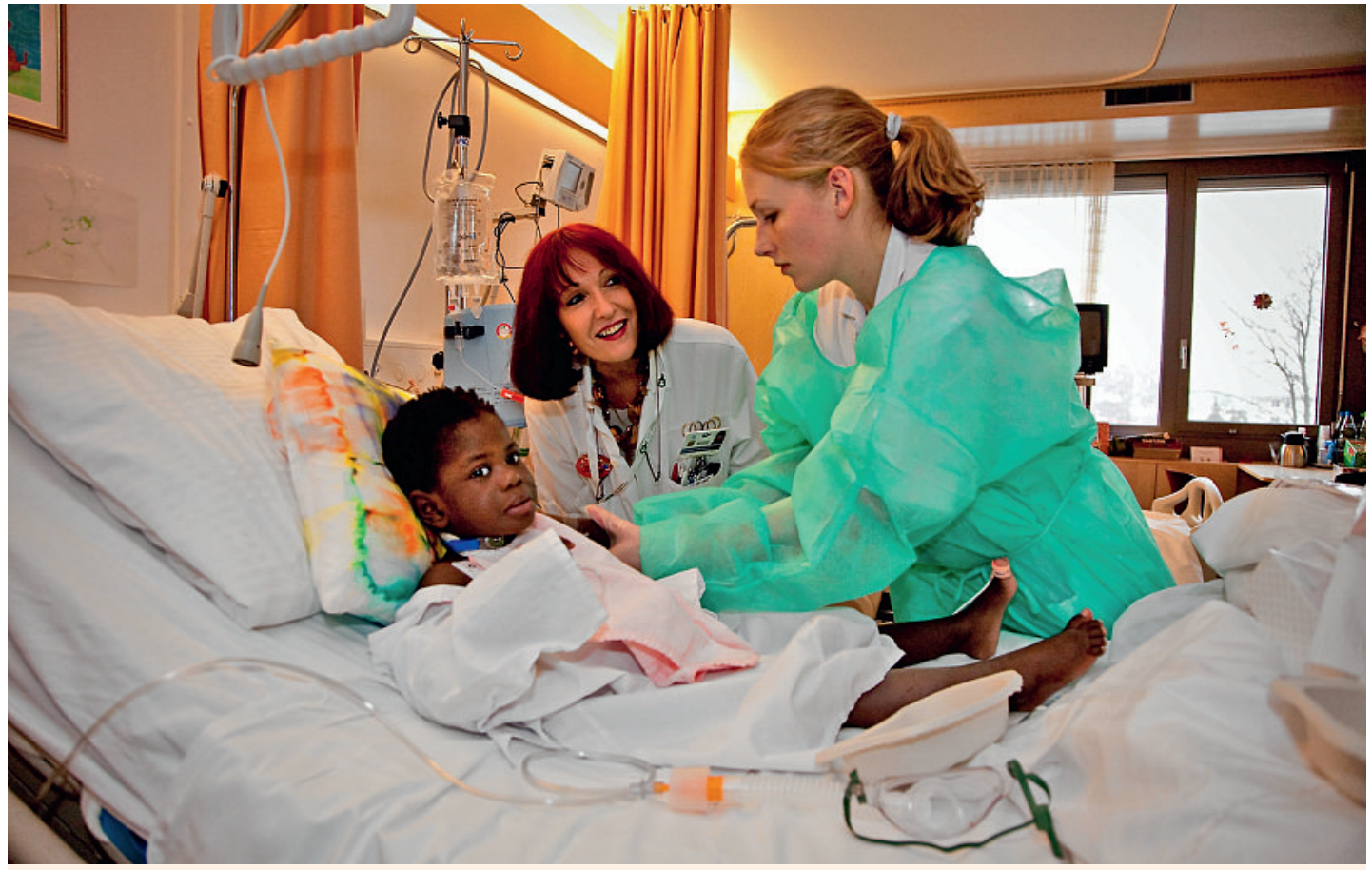

Nur wenn fehlende Geräte oder mangelndes Know-how eine Operation in Afrika unmöglich machen, werden die Patienten nach Europa, hier ins CHUV Lausanne, überwiesen. 
jektländern. Schweizer Ärzte und anderes medizinisches Personal haben jeweils zeitlich begrenzte Einsätze in der Dritten Welt. Sie geben ihr Wissen mittels Kursen, Konferenzen und Vorträgen weiter, führen aber auch schwierige Operationen vor Ort durch. Des weiteren arbeiten sie mit den Verantwortlichen der Gesundheitspolitik zusammen. Dies soll eine nachhaltige Verbesserung der Gesundheitsstrukturen zur Folge haben. Zusätzlich bieten Schweizer Spitäler Weiterbildungskurse für medizinisches Personal aus den Projektländern an. So reisen jährlich Ärzte und Krankenschwestern aus diesen Ländern in die Schweiz, um sich fortzubilden.

In der Schweiz arbeitet Terre des hommes Kinderhilfe für «Voyage vers la vie» mit den Universitätsspitälern Genf (HUG) und Lausanne (CHUV) zusammen. Professor von Segesser operierte im Rahmen des Projektes bereits Anfang der 80er Jahre in Genf Kinder mit Herzproblemen. Als Abteilungsleiter der Kardiovaskulären Chirurgie am Universitätsspital Lausanne führt er diese Tätigkeit weiter. Neben den Operationen, die er in der Schweiz durchführt, nahm von Segesser auch an Kurzeinsätzen im Ausland teil. Während zehn Jahren besuchte er Krankenhäuser in verschiedenen Ländern, so unter anderem in Tunesien und Ägypten.

\section{Zahlreiche Hürden sind zu überwinden}

Werden die Patienten in die Schweiz überwiesen, werden sie von Beginn weg im Haus Massongex im Wallis beherbergt und betreut. Bereits im Projektland selbst wird eine möglichst präzise Diagnose gestellt, um die Voraussetzungen für eine erfolgreiche Behandlung zu optimieren. Doch oft fehlen Know-how und medizinische Geräte für eine definitive Diagnose vor Ort. Der Patientenfall wird in der Schweiz deshalb einem Komitee, bestehend aus allen involvierten Ärzten, vorgelegt und gemeinsam wird die sinnvollste Behandlung besprochen. Da die Kinder nur für kurze Zeit in der Schweiz weilen, würden nicht immer Standardverfahren angewendet, erzählt von Segesser. Denn wird bei Kindern mit Herzproblemen künstliches Material eingesetzt, wäre eine notwendige Folgeoperation programmiert, was nicht im Sinne des Projekts wäre. Ein weiterer Unterschied sei, dass die angeborenen Herzprobleme in der Schweiz meist bereits pränatal diagnostiziert werden. Der Grossteil der herzgeschädigten Kinder, die in der Schweiz aufwachsen, kann deshalb noch im ersten Lebensjahr operiert werden. Dies ist bei den Patienten des Projektes natürlich nicht möglich, da die Diagnosemöglichkeiten in der Dritten Welt nicht in diesem Masse gewährleistet sind. Oft würden die Diagnosen gar zufällig im Rahmen der Behandlung anderer Leiden gestellt, berichtet der Chirurg. Meist sind die Kinder dann bereits 5 oder 6 Jahre alt, was zur Folge hat, dass die Schäden bei angeborenen Herzfehlern zum Teil schon ein irreversibles Ausmass angenommen haben.

\section{Prof. Ludwig K. von Segesser}

Professor von Segesser ist in Luzern aufgewachsen und absolvierte sein Medizinstudium an der Universität Basel. Anschliessend war er am Kantonsspital Obwalden (Sarnen) und im Universitätsspital Genf tätig. In letzterem war er Oberarzt der Herzchirurgie. Daraufhin arbeitete er in den USA (Texas Heart Institute in Houston) und am Universitätsspital Zürich. Seit 1996 ist Professor von Segesser am Universitätsspital in Lausanne Abteilungsleiter der Kardiovaskulären Chirurgie und zusätzlich amtiert er als Präsident der Schweizerischen Herzstiftung.

\section{Stiftung Terre des hommes - Kinderhilfe}

Terre des hommes - Kinderhilfe (Tdh) ist ein international tätiges Schweizer Kinderhilfswerk. Jährlich unterstützt Tdh über eine Million Kinder und Angehörige in mehr als 30 Ländern. Die Stiftung wurde 1960 von Edmond Kaiser gegründet. Sie zählt heute weltweit über 1300 Mitarbeiter und wird zusätzlich von mehr als 2000 freiwilligen Helfern in der Schweiz unterstützt. Weitere Informationen finden Sie unter www.tdh.ch

Besteht jedoch eine grosse Chance auf Heilung, wird der Patient nach Europa überwiesen.

\section{Erfolg zu 100 Prozent ist nie garantiert}

Null Risiko gebe es nicht, hält Professor von Segesser im Bezug auf die genannten Hürden fest. Er darf bei den Operationen auf eine 98-prozentige Erfolgsquote blicken. Wegen der zahlreichen Hindernisse, welche die Ärzte des Projektes bewältigen müssen, sterben aber trotz aller Anstrengungen zwei Prozent der Patienten auf dem Operationstisch oder an den Folgen der Behandlung, was ungefähr der Quote bei Schweizer Patienten entspricht. Von Segesser begründet dies damit, dass es bei den Patienten des Projekts zwar kaum Notfälle gibt, die bei Eingriffen naturgemäss höheren Risiken ausgesetzt sind. Dagegen sind die Kinder aus den Drittweltländern geschwächter und die Probleme sind weiter fortgeschritten. Deshalb gleicht sich die Erfolgs- bzw. Misserfolgsquote in etwa aus.

Von Segesser meint, dass er fast ausschliesslich rührende Momente mit dem Projekt erlebe. Ein Mädchen blieb ihm besonders in Erinnerung: Die Operation der kleinen Patientin musste aus organisatorischen Gründen verschoben werden. Das Kind war dermassen entsetzt darüber, dass es sich vor die Tür des Operationssaales setzte, bittere Tränen weinte und nur mit Müh und Not davon überzeugt werden konnte, wieder ins Bett zu gehen. Es hatte Angst, dass es die lebensrettende Operation doch nicht erhalten würde. Zum Schluss ging aber alles gut, und seine Reise in die Schweiz wurde zur Reise (zurück) ins Leben. 